\title{
OS RISCOS ENFRENTADOS PELOS PROFISSIONAIS DE ENFERMAGEM NO EXERCÍCIO DA ATIVIDADE LABORAL
}

\author{
Silvani Neves Paiva de Araújo* \\ Autor correspondente: Silvani Neves Paiva de Araújo - silvanidearaujo@yahoo.com.br, \\ * Especialista em Docência e Metodologia de Ensino Superior, Salvador, Bahia, Brasil..
}

\begin{abstract}
Resumo
Os riscos ocupacionais estão presentes constantemente no cotidiano dos profissionais de enfermagem através de situações que podem ocasionar o desequilíbrio físico, mental e social dos mesmos. O estudo teve como objetivo compreender e evidenciar os riscos enfrentados pelos profissionais de enfermagem na realização do processo de cuidar, verificando os desgastes sofridos por estes impactos na saúde destes trabalhadores ao exercer sua atividade laboral. Trata-se de uma revisão sistemática com revisão da literatura. Obteve como resultado que diversas cargas e desgastes conduzem a problemas na saúde destes funcionários, bem como alto índice de absenteísmo.
\end{abstract}

Palavras-chave: Saúde. Riscos. Agravos. Saúde do trabalhador. Enfermagem.

\begin{abstract}
Occupational hazards are present constantly in everyday nursing professionals through situations that can cause physical, mental and social imbalance thereof. The study aimed to understand and highlight the risks faced by nurses in implementing the care process, checking the wear suffered by these impacts on their health by exercising their labor activity. This is a systematic review with literature review. He found that several loads and wear lead to health problems such officials as well as high rate of absenteeism.
\end{abstract}

Kepwords: health; risks; injuries; occupational health; nursing. 


\section{INTRODUÇÃO}

No exercício da atividade laboral, os trabalhadores de enfermagem estão propensos a sofrerem acidentes de trabalho devido a diversas cargas causadas por fatores físicos, químicos, fisiológicos, psíquicos, mecânicos e principalmente os biológicos, que podem ocasionar doenças ocupacionais. Esta situação relaciona-se ao fato do cuidado direto $\mathrm{e}$ indireto que realizam aos pacientes e, também, devido aos tipos e frequência dos procedimentos que executam. ${ }^{(1)}$

Constatada a necessidade de humanizar este trabalho para obter, consequentemente, um bom atendimento aos pacientes, objeto de sua responsabilidade, é indispensável uma atenção especial à sua própria saúde, que precisa ser valorizada. Sendo assim, o sistema de saúde tem demonstrado tardiamente seu interesse pelos temas referentes às cargas de trabalho, obrigações e riscos a que estão expostos estes trabalhadores, bem como as atividades realizadas em função daqueles que recebem seus cuidados. ${ }^{(2)}$

Neste contexto, em 11 de novembro de 2005, por meio da portaria $\mathrm{GM} \mathrm{n} \mathrm{n}^{\circ} 85$ foi regulamentada a norma 32 com o objetivo de propor uma legislação específica que pudesse implementar medidas de proteção a segurança e saúde dos trabalhadores dos serviços de saúde bem como daqueles que exercem atividades de promoção e assistência a saúde em geral.(3)

Em sua maioria, as cargas de trabalho a que são expostos os profissionais de saúde constituem-se em elementos, os quais se traduzem num processo de desgaste evidenciado pela perda total ou parcial das capacidades corporal e psíquica, abrangendo os processos biopsíquicos em seu conjunto e, logo, demonstram as características da sociedade e definem o perfil patológico do grupo específico. ${ }^{(4)}$

É visível que o profissional de enfermagem é imprescindível para a vida humana, por se tratar de elemento fundamental no zelo pelo bem estar e saúde dos pacientes. Entretanto, questiona-se quem é responsável por zelar pela saúde desses profissionais que, em determinadas situações, descuidam-se do seu próprio autocuidado. Desse modo, em 22 de dezembro de 2009, a Vigilância em Saúde do Trabalhador (VISAT), por meio da Portaria GM/MS $n^{\circ} 3252$, aprova as diretrizes para a execução e financiamento das ações de Vigilância em Saúde e dispõe sobre a promoção da saúde e a redução da morbimortalidade da população trabalhadora, por meio da integração de ações que intervenham nos agravos e seus determinantes decorrentes dos modelos de desenvolvimento e processo produtivos. ${ }^{(5)}$

Assim, é possível evidenciar através do fundamento teórico fornecido a partir dos autores supracitados, que o cumprimento do trabalho de enfermagem pode trazer consequências para a saúde do trabalhador impactando na sua qualidade de vida e na assistência prestada. Diante desta consideração, surge o interesse em desenvolver uma revisão sobre o tema, contribuindo na disseminação do conhecimento adquirido para os profissionais da saúde, viabilizando a realização de ações que possam promover e prevenir a saúde destes profissionais, minimizando os riscos que possam comprometer a integridade física e mental dos mesmos.

Por conseguinte, o objetivo deste estudo é evidenciar e compreender os riscos enfrentados pelos profissionais de enfermagem na realização do processo de cuidar, verificando os desgastes ocorridos e os impactos causados na saúde destes trabalhadores ao exercer sua atividade laboral.

\section{METODOLOGIA}

Trata-se de um estudo de revisão sistemática da literatura, acerca dos riscos enfrentados pelos profissionais de enfermagem na realização de seu ofício durante o processo de cuidar, constatando, assim, o conhecimento produzido sobre este tema e obtendo elementos para analisá-lo.

Uma revisão sistemática, assim como outros tipos de estudo de revisão, é uma forma de pesquisa que utiliza como fonte de dados a literatura so- 
bre determinado tema. Esse tipo de investigação disponibiliza um resumo das evidências relacionadas a uma estratégia de intervenção específica, mediante a aplicação de métodos explícitos e sistematizados de busca, apreciação crítica e síntese da informação selecionada. ${ }^{(6)}$

Este artigo procurou responder a pergunta que sustenta esta pesquisa: podem os profissionais de enfermagem sofrer agravos durante o exercício de sua atividade laboral?

A procura por artigos incluiu pesquisas em bases eletrônicas. As bases pesquisadas foram: Literatura Latino-Americana e do Caribe em Ciências da Saúde (LILACS); Scientific Eletronic Library (SciELO) e Medical Literature Analysisand Retrieval System Online (MEDLINE). Para a busca dos artigos selecionados foram utilizadas palavras-chaves em português, perante consulta aos Descrito- res em Ciências da Saúde (DeCS): riscos, agravos, cuidar, saúde do trabalhador e enfermagem. Utilizando como base estes descritores foram encontradas 95 publicações, sendo considerados apenas uma vez aqueles duplicados.

Para selecionar os artigos, inicialmente, realizou-se a leitura das 95 publicações com a meta de apurar a amostra por meio de critérios de inclusão e exclusão. Foram incluídos artigos originais completos, publicados no período compreendido entre os anos de 2005 e 2013 , em periódicos nacionais de enfermagem, com qualificação nacional e internacional do sistema de classificação da CAPES. Excluíram-se os artigos de revisões sistemáticas, assim como os de revisão integrativa, materiais em línguas estrangeiras e teses de doutorado. Desta forma, a amostra final foi constituída por 20 artigos como demonstrado abaixo.

Tabela 1 - Distribuição de artigos identificados e selecionados segundo bases de dados

\begin{tabular}{cccc}
\hline BASE DE DADOS & $\begin{array}{c}\text { ARTIGOS } \\
\text { IDENTIFICADOS }\end{array}$ & $\begin{array}{c}\text { ARTIGOS PRÉ- } \\
\text { SELECIONADOS }\end{array}$ & $\begin{array}{c}\text { ARTIGOS } \\
\text { SELECIONADOS }\end{array}$ \\
\hline LILACS & 27 & 8 & 3 \\
SCIELO & 68 & 28 & 17 \\
MEDLINE & 0 & 0 & 0 \\
TOTAL & 95 & 36 & 20 \\
\hline
\end{tabular}

Fonte: Elaborado pelas autoras

\section{RESULTADOS E DISCUSSÃO}

Os 20 artigos utilizados para embasamento deste trabalho de revisão sistemática permitiu realizar uma análise dos dados, no quais foi possível identificar os processos de desgaste e as formas de trabalho ocorridas pela exposição dos profissionais às cargas químicas e biológicas a que estão submetidos durante a realização de sua atividade laboral.

De acordo com o ano de publicação, 2009 esteve em constante evidência, com cinco artigos publicados. A Revista Latino-Americana de Enfermagem (Rev. Latino-Am. Enfermagem) foi o periódico com maior número de artigos utilizados, totalizando sete.

Analisando as questões de autoria, a maioria dos artigos foi construído em parceria por dois autores, ${ }^{(11)}$ seguido pela construção por mais de dois autores. ${ }^{(8)}$ Ainda em relação a este item, os 20 artigos totalizam 59 autores diferentes, sendo em sua maioria elaborados por profissionais de enfermagem.

As produções integrantes deste estudo estão expostas em tabela, na qual são identificados o título, autores, ano de publicação, objetivos, métodos e materiais, resultado e conclusão. 
Dentre os resultados apresentados pelos estudos utilizados como embasamento, constatou que o trabalho desenvolvido por estes funcionários muitas vezes em situação rotineira, mecânica e repetitiva, pode comprometer a sua saúde gerando processos de desgaste físicos os quais podem acarretar a perda ou a redução da capacidade de produzir, bem como o agravamento e aparecimento de doenças como as lesões osteoarticulares e outros agravos à saúde. Devido às cargas de trabalho, estes profissionais sofrem processos de desgaste, resultantes da exposição constante aos riscos e são demonstrados na somatização de algumas experiências (manifestadas de forma física ou psicológica) ou pelas desordens psicoemocionais (sinais de angústia, falta de motivação, medo etc.). (4-7)

Existem diversos fatores que afetam a saúde dos profissionais de enfermagem. Estes que atuam dentro de unidade hospitalar estão expostos a cargas que são geradoras de processos de desgastes; dentre as cargas apresentadas, as mais expressivas foram as mecânicas e fisiológicas. ${ }^{(7-8)}$

No ambiente de trabalho os fatores ecológicos e de ambientação também contribuem para o desgaste físico destes profissionais. Assim, o controle das condições de trabalho influencia na qualidade, na saúde física e bem estar do trabalhador em seu local de trabalho. Devido ao exercício da atividade laboral, os profissionais de enfermagem são considerados os mais propícios para sofrerem acidentes de trabalho, os quais, em sua maioria, ocorrem durante a realização de procedimentos. Em determinados momentos o descarte ou acondicionamento inadequado dos resíduos também geram acidentes, em alguns casos causados pelas agulhas. ${ }^{(9-1-10)}$

É possível dizer que estes profissionais estão constantemente expostos a riscos, o que afeta suas condições de saúde. Muitos profissionais acreditam que os riscos ocupacionais psicossociais são os que mais os acometem. Dentre eles, destacam-se as agressões físicas e verbais; a falta de segurança no trabalho e um ambiente de trabalho estressante. Outro fator que causa desgaste é a exposição cotidiana às cargas psíquicas. ${ }^{(1-4)}$
Analisando as cargas psíquicas é possível evidenciar que estas são atribuídas a algumas condições de trabalho, além de serem potencializadoras e potencializadas pela maioria das cargas. Assim, as cargas fisiológicas são identificadas e percebidas pelos profissionais de enfermagem como elemento presente na realidade do trabalho; a exposição fisiológica gera desgaste da força de trabalho, que pode ser apreendida por sinais/sintomas que interferem na qualidade de vida e laboral.(4-12)

As cargas mecânicas são reconhecidas como acidentes de trabalho por provocarem lesões que consistem na ruptura de segmentos do corpo como, por exemplo, as contusões, fraturas, perfurações, cortes e hematomas. Além dos ferimentos perfuro cortantes, potencializados pelas cargas biológicas, pela capacidade em contaminar os trabalhadores com vírus importantes e letais, como o da Hepatite $\mathrm{B}$ e $\mathrm{C}$ e o vírus da Síndrome da Imunodeficiência Adquirida (AIDS). Os trabalhadores registram e buscam registrar esta exposição muitas vezes pelo medo e o comprometimento com sua saúde. ${ }^{(8)}$

Além dos desgastes pelos quais os profissionais de enfermagem passam em seu cotidiano, ainda em algumas instituições existem condições de trabalho precárias, que somadas a sua qualidade de vida aumentam a possibilidade de adoecimento. Somado a isto existem ainda pessoal não treinado, a escassez de recursos materiais e humano; modelo de gestão centralizador; proximidade com a morte e conflitos no trabalho que fazem com que o trabalhador sinta-se insatisfeito, ocasionando fadiga mental e física. ${ }^{(13-14-15)}$

Por meio de estudos realizados em trabalhadores de enfermagem em 23 instituições de saúde (do estado de Minas Gerais) atendidos pela Divisão de Assistência à Saúde do Trabalhador; entre os 6.070 atendimentos realizados aos profissionais de enfermagem, $11,83 \%$ deles ( 718$)$ apresentaram diagnósticos de problemas relacionados ao sistema osteomuscular, envolvendo diversas estruturas corporais como a coluna vertebral, membros superiores e inferiores. ${ }^{(16-11)}$ 
As doenças legalmente consideradas como doenças do trabalho relacionadas ao sistema músculo - esquelético foram identificadas em 255 (35\%) atendimentos, destacando-se as dorsalgias (20\%) e as sinovites e tenossinovites (13,7\%); constatou-se, ainda, que, dos 862 atendimentos psicológicos realizados, $473(54,9 \%)$ foram destinados aos trabalhadores de enfermagem. Os elevados índices de atendimentos médico ( $43 \%)$ e psicológico $(54,9 \%)$ prestados aos trabalhadores de enfermagem chamam a atenção. Posteriormente, em estudo realizado em duas unidades de urgência e emergência, também no estado de Minas Gerais, foi constatado que os riscos ocupacionais mais encontrados foram os psicossociais; agressões físicas e verbais $(63,53 \%)$, falta de segurança no trabalho $(14,12 \%)$ e ambiente de trabalho estressante (10.58\%). ${ }^{(16-11)}$

Muitos destes fatores apresentados contribuem para que haja o desenvolvimento da Síndrome de Burnout, que decorre de um processo gradual de desgaste no humor e desmotivação acompanhado de sintomas físicos e psíquicos. O trabalhador perde o sentido da sua relação com o trabalho e faz com que as coisas já não tenham mais importância. É caracterizada por três dimensões sintomatológicas: exaustão emocional verificada pela presença do esgotamento emocional e/ou físico, despersonalização observada pela insensibilidade emocional ou endurecimento afetivo e falta de envolvimento no trabalho, identificada pela inadequação pessoal e profissional. A situação fica mais explícita no estudo realizado com cinquenta e três trabalhadores, no qual o estresse aparece em 36 trabalhadores, dos quais $43,4 \%$ responderam que ele foi provocado pelas condições de trabalho e 22,6\% afirmam ter sido agravado; 29 convivem com a mudança de humor, dos quais $26,4 \%$ creditam ao trabalho esta alteração, enquanto $22,6 \%$ consideram que a atividade laboral apenas agrava a mudança de humor. ${ }^{(17-02)}$

Os profissionais de enfermagem estão expostos a condições de trabalho diversas que incluem longas jornadas de trabalho em turnos desgastantes, nos rodízios de setores, em multiplicidade, repeti- tividade e monotonia de funções, intensividade e ritmo excessivo de trabalho, ansiedade, esforços físicos, posições incômodas, na separação do trabalho intelectual e manual e no constante controle das chefias. Desta forma, é possível dizer que estes profissionais atuam na interface do processo de adoecimento da população e das doenças relacionadas ao trabalho. Ali se evidenciam doenças crônicas que interferem no processo de trabalho. ${ }^{(18-19)}$

Como consequência destes desgastes sofridos pelos profissionais de enfermagem, existe um grande número de absenteísmo decorrente do afastamento para tratar de doenças originadas ou desencadeadas pelo processo diário no cumprimento do seu dever. Um dos acontecimentos mais frequente de adoecimento entre os profissionais da área da saúde está relacionado ao sistema osteomuscular e osteoarticular. Sendo assim, a adoção de medidas que visem à redução das demandas psicológicas proveniente do trabalho, autonomia ao trabalhador, maior atenção às posturas adotadas na execução das atividades laborais, condições de mobiliários, disponibilizar instrumentos e equipamentos ergonomicamente planejados são algumas das atitudes que podem e devem ser tomadas para reduzir este quadro. (04-20-16-18)

Mediante pesquisa realizada em hospital de Ensino de Curitiba, pode-se evidenciar que dos afastamentos ocorridos, o maior número foi relativo a licenças médicas, 96 casos, representando $75,0 \%$. Destes, 32 (25,2\%) foram causados principalmente por doenças do sistema osteoarticular; 27 (21,3\%) foram consequências de causas externas (traumas); $26(20,5 \%)$ correspondem a transtornos mentais e comportamentais; 13 (10,2\%) devido a doenças do aparelho respiratório e 07 (5,5\%) por doenças do aparelho circulatório. Em outro estudo realizado no hospital de ensino na região Centro-Oeste, as doenças do sistema osteomuscular e do tecido conjuntivo representam o principal problema de saúde, correspondendo cerca de $21 \%$ das notificações, seguido por transtornos mentais e comportamentais $(15,3 \%)$ e problemas respiratórios $(13,9 \%) .{ }^{(08-21)}$ 
As doenças osteomusculares consideradas como relacionadas ao trabalho podem ser enquadradas como pertencentes ao grupo das lesões por esforços repetitivos (LER), uma vez que tal terminologia indica somente que os diagnósticos dessas afecções ou síndromes estão relacionadas com as condições do trabalho do indivíduo. Trata-se de uma síndrome clínica caracterizada por dor crônica, acompanhada ou não de alterações objetivas, que se manifesta, principalmente, no pescoço, cintura escapular e/ou membros superiores, em decorrência do trabalho, podendo afetar tendões, músculos e nervos periféricos. Sob aquelas denominações, abrigam-se várias afecções dos tecidos musculoesqueléticos, objetos de várias especialidades médicas como a ortopedia, reumatologia e a neurologia, entre outras. Por meios dos estudos constatou que a maioria dos profissionais de enfermagem trabalha em turnos duplos ou triplos, refletindo em algumas horas para o lazer, descanso, exercício físico, qualidade do sono, além de aumento da exposição às cargas de trabalho e os riscos presentes em seus ambientes de trabalho. ${ }^{(8-22)}$

Outrossim, é necessário que seja ressaltado que trabalhadores de enfermagem estão propícios a acidentes de trabalho por exercem sua atividade em ambiente que oferece riscos biológicos, químicos e físicos. Uma importante medida para impedira ocorrência de novos acidentes seria a prevenção dos erros humanos, mediante adoção de treinamento contínuo dos profissionais e uso de óculos de proteção. ${ }^{(9)}$

Assim, o processo que ocorre no ambiente de trabalho dos profissionais de enfermagem onde são expostos as diversas cargas e o desenvolvimento do desgaste ocasiona em impactos na saúde física, psíquica e emocional do indivíduo, envolvendo sua racionalidade, bem-estar social e saúde física, devido à somatização. Em consonância com os resultados da pesquisa, outros estudos demonstram que as taxas de absentismo relacionado à doença em enfermagem é muito elevado, principalmente devido à presença de fatores de risco no ambiente de trabalho. Em consenso com os resultados da pes- quisa, é possível observar que as taxas de absentismo relacionado à doença em enfermagem são muito elevados, principalmente devido à presença de fatores de risco no espaço de trabalho. ${ }^{(4-21)}$

\section{CONSIDERAÇÕES FINAIS}

Esse estudo possibilitou observar que o ambiente de trabalho propicia diversos riscos à saúde dos profissionais de enfermagem, que podem ser evitados ou reduzidos por meio de medidas de proteção variadas. Devido ao desconhecimento e a não identificação de determinadas situações de risco, o trabalhador pratica ações sem equipamentos de proteção individual, o que pode acarretar em acidentes do trabalho ou doenças ocupacionais. Os acidentes ou doenças a que são acometidos, além de impedir, temporária ou permanentemente, o profissional de exercer seu trabalho por alterações físicas, acarreta em transtornos psíquicos ou emocionais importantes. Vale ressaltar que estes trabaIhadores estão sujeitos aos acidentes de trabalho porque o ambiente profissional oferece riscos biológicos, químicos e físicos entre outros.

O trabalho desenvolvido em condições atribuladas, acompanhado da desvalorização profissional, remuneração inadequada, relações hierárquicas e interpessoais conflituosas em seu ambiente de trabalho provoca a insatisfação do trabalhador, o que favorece o aparecimento do estresse neste indivíduo. Em decorrência destes fatores somados às cargas enfrentadas pelos profissionais de enfermagem originam-se elevados índices de absenteísmo.

Assim, pode-se dizer que estes profissionais de enfermagem apresentam diversos fatores que são desencadeadores para o aparecimento de doenças ocupacionais. Faz-se importante repensar a estrutura organizacional dos serviços de saúde sob a perspectiva do trabalhador que, efetivamente, é delimitado cotidianamente com as cargas de trabalho, devendo ser o ponto focal de propostas intervencionistas para as instituições de saúde. 


\section{REFERÊNCIAS}

1. Balsamo AC, Felli VEA. Estudo sobre os acidentes de trabalho com exposição aos líquidos corporais humanos em trabalhadores da saúde de um hospital universitário. Rev. latinoam. enferm. 2006;14(3):346-353.

2. Mauro MY, Veiga A. Problemas de saúde e riscos ocupacionais: percepções dos trabalhadores de enfermagem de unidade materno infantil. Rev. enferm. UERJ. 2008;16(1):68-9.

3. Brasil. Portaria $n^{\circ} 485$, de 11 de novembro de 2005. Aprova a Norma Regulamentadora n 32 (Segurança e Saúde no Trabalho em Estabelecimentos de Saúde). [internet]. Brasília, DF; 2005. Disponível em: http://www.camara. gov.br/sileg/integras/726447.pdf

4. Mininel VA et al. Cargas psíquicas e processos de desgaste em trabalhadores de enfermagem de hospitais universitários brasileiros. Rev. latinoam. enferm. 2011;19(O2):01-09.

5. Brasil. Portaria $\mathrm{n}^{\circ}$ 3.252, de 22 de dezembro de 2009. Aprova as diretrizes para execução e financiamento das ações de Vigilância em Saúde pela União, Estados, Distrito Federal e Municípios e dá outras providências. [internet]. Brasília, DF; 2OO9. Disponivel em: http://www. saude.mg.gov.br/images/documentos/Portaria_ GM_3252_de_22_dezembro_de_2009.pdf

6. Sampaio RF, Mancini MC. Estudo de revisão sistemática: um guia para síntese criteriosa da evidência científica. Rev. bras. fisioterap. 2007;11(1):83-89.

7. Santana LL, Miranda FMD, Karino ME, Baptista PCP, Felli VEA, Sarquis LMM. Cargas e desgastes de trabalho vivenciados entre trabalhadores de saúde em um hospital de ensino. Rev. gaúch. enferm. 2013;34(1):64-70.

8. Costa T, Felli VE. Exposição dos trabalhadores de enfermagem às cargas químicas de um hospital público universitário da universidade de São Paulo. Rev. latinoam. enferm. 2005;13(4):5001-8.

9. Almeida $C$ et al. Acidentes de trabalho envolvendo os olhos: avaliação de riscos ocupacionais com trabalhadores de enfermagem. Rev. latinoam. enferm. 2005;13(05):708-716.
10. Almeida C, Benatti MC. Exposições ocupacionais por fluidos corpóreos entre trabalhadores da saúde e sua adesão à quimioprofilaxia. Rev. Esc. Enferm. USP. 2007; $41(\mathrm{OI}): 12 \mathrm{O}-6$.

11. Dalrí R et al. Riscos ocupacionais e alterações de saúde entre trabalhadores de enfermagem brasileiros de unidades de urgência e emergência. Cienc. enferm. 2010;16(2): 69-81.

12. Sápia T et al. Problemas de saúde de trabalhadores de enfermagem em ambulatórios pela exposição à cargas fisiológicas. Acta paul. enferm. 2009;22(6):808-13.

13. Elias MA, Navarro VL. Relação entre o trabalho, a saúde e as condições de vida: negatividade e positividade no trabalho das profissionais de enfermagem de um hospital escola. Rev. latinoam. enferm. 2006.14(4):517-25.

14. Hanzelmann R, Passos J. Imagens e representações da enfermagem acerca do stresse sua influência na atividade laboral. Rev. Esc. Enferm. USP. 2010;44(3):694-701.

15. Azambuja EP et al. É possível produzir saúde no trabalho da enfermagem. Rev. Texto \& contexto enferm. 2010;19(4): 658-66.

16. Murofuse N, Marziale MH. Doenças do sistema osteomuscular em trabalhadores de enfermagem. Rev. latinoam. enferm. 2005;13(O3):364-373.

17. Jodas D, Haddsad MC. Síndrome de Burnout em trabalhadores de enfermagem de um pronto socorro de hospital universitário. Acta paul. enferm. 2009;22(O2):192-197.

18. Abreu R, Simões A. Ausências por adoecimento na equipe de enfermagem de um hospital de ensino. Ciênc. cuid. Saúde. 2009; 8(4):637-644.

19. Teixeira RC, Mantovani MF. Enfermeiros com doenças crônicas: as relações com o adoecimento, à prevenção e o processo de trabalho. Rev. Esc. Enferm. USP. 2009;43(2):415-421.

2O. Magnago TS et al. Condições de trabalho de profissionais da enfermagem: avaliação baseada no modelo demanda controle. Acta paul. enferm.2010;23(6):811-817.

21. Mininel VA et al. Cargas de trabalho, processos de desgaste e absenteísmo-doença em enfermagem. Rev. latinoam. enferm. 2013;21(6):1290-1297. 